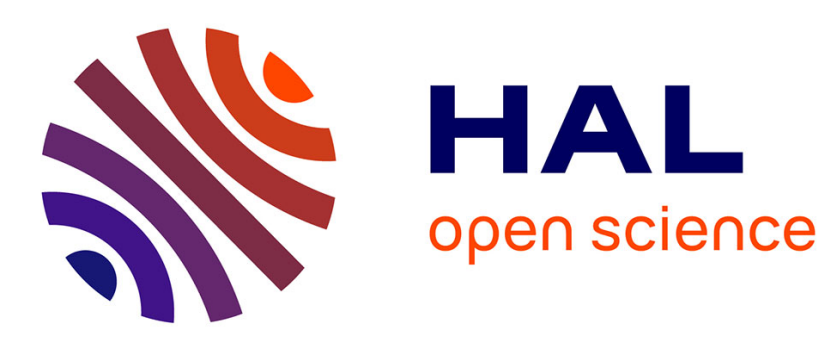

\title{
En marge des invasions vikings: Actard de Nantes et les translations d'évêques propter infestationem paganorum
}

Pierre Bauduin

\section{To cite this version:}

Pierre Bauduin. En marge des invasions vikings: Actard de Nantes et les translations d'évêques propter infestationem paganorum. Le Moyen Age. Revue d'histoire et de philologie, 2011, 117 (1), pp.9-20. 10.3917/rma.171.0009 . hal-00651872

\section{HAL Id: hal-00651872 \\ https://hal.science/hal-00651872}

Submitted on 1 Apr 2020

HAL is a multi-disciplinary open access archive for the deposit and dissemination of scientific research documents, whether they are published or not. The documents may come from teaching and research institutions in France or abroad, or from public or private research centers.
L'archive ouverte pluridisciplinaire HAL, est destinée au dépôt et à la diffusion de documents scientifiques de niveau recherche, publiés ou non, émanant des établissements d'enseignement et de recherche français ou étrangers, des laboratoires publics ou privés. 


\title{
EN MARGE DES INVASIONS VIKINGS : ACTARD DE NANTES ET LES TRANSLATIONS D'ÉVÊQUES PROPTER INFESTATIONEM PAGANORUM
}

\author{
Pierre Bauduin
}

De Boeck Supérieur | «e Moyen Age »

2011/1 Tome CXVII | pages 9 à 20

ISSN 0027-2841

ISBN 9782804165369

Article disponible en ligne à l'adresse :

https://www.cairn.info/revue-le-moyen-age-2011-1-page-9.htm

Distribution électronique Cairn.info pour De Boeck Supérieur.

(C) De Boeck Supérieur. Tous droits réservés pour tous pays.

La reproduction ou représentation de cet article, notamment par photocopie, n'est autorisée que dans les limites des conditions générales d'utilisation du site ou, le cas échéant, des conditions générales de la licence souscrite par votre établissement. Toute autre reproduction ou représentation, en tout ou partie, sous quelque forme et de quelque manière que ce soit, est interdite sauf accord préalable et écrit de l'éditeur, en dehors des cas prévus par la législation en vigueur en France. Il est précisé que son stockage dans une base de données est également interdit. 


\section{En marge des invasions vikings: Actard de Nantes et les translations d'évêques propter infestationem paganorum ${ }^{*}$}

L'évêque d'une cité menacée ou détruite par les païens peut-il obtenir d'être transféré vers un autre siège épiscopal? La question revêt, dans la seconde moitié du Ix ${ }^{\mathrm{e}}$ siècle, une importance que révèlent plusieurs affaires où sont directement allégués les périls encourus par des prélats en raison des attaques des Vikings. Vers 861, Hunfrid évêque de Thérouanne, avait demandé à Nicolas $\mathrm{I}^{\mathrm{er}}$ si, chassé de son évêché par les Normands, il devait désormais vivre dans un monastère ${ }^{1}$. En réponse, le pape avait rappelé le devoir, pour l'évêque, de rester parmi ses brebis, puis de rassembler son troupeau une fois passée la tourmente. De fait, la situation de Hunfrid était moins périlleuse qu'il ne semble et l'argument cachait plutôt l'insatisfaction de l'évêque dans l'exercice de sa charge épiscopale ${ }^{2}$. Le dossier le plus complexe est cependant celui d'Actard, évêque de Nantes, transféré vers une autre Église en raison du «voisinage»- le terme est repris dans plusieurs lettres - incommode non seulement des Normands mais aussi des Bretons, à un moment où le roi Salomon tente d'affranchir le Bretagne de sa métropole tourangelle pour obtenir l'autonomie du clergé breton ${ }^{3}$. Il existe, sur ce transfert, une documentation importante, principalement livrée par la Chronique

* Auteur: Pierre Bauduin, Université de Caen Basse-Normandie, UMR 6273 CRAHAM, pierre.bauduin@unicaen.fr.

1. Nicolas I ${ }^{\text {er }}$ Epistolae, éd. E. Perels, Epistolae Karolini Aevi, t. 4, M.G.H., Epistolae, t. 6, Berlin, 1925, p. 612, n 104 : Sciscitaris itaque per Guandalgarium presbyterum, utrum, cum a Normannis de episcopio depelleris, debeas de cetero in monasterio conversari.

2. C. Mériaux, Thérouanne et son diocèse jusqu'à la fin de l'époque carolingienne. Les étapes de la christianisation d'après les sources écrites, Bibliothèque de l'École des chartes, t. 158, 2000, p. 403; ID., Gallia irradiata. Saints et sanctuaires dans le nord de la Gaule du haut Moyen Âge, Stuttgart, 2006, p. 160.

3. A. Chédeville, H. Guillotel, La Bretagne des saints et des rois, $v^{e}-X^{e}$ siècle, Rennes, 1984, p. 304-313.

DOI : 10.3917/ rma.162.0009 
de Nantes ${ }^{4}$, une œuvre tardive et portée à justifier l'annexion de la région de Guérande au début du $\mathrm{XI}^{\mathrm{e}}$ siècle ${ }^{5}$, et un ensemble de lettres pour la plupart datées entre 866 et $872^{6}$, parmi lesquelles figure une réponse de Hincmar à la consultation d'un évêque. Sur un certain nombre de points ce texte contredit $\mathrm{d}^{\prime}$ autres pièces du dossier et expose longuement la position canonique de l'archevêque relativement à Actard, investi de l'Église de Tours (871) tout en conservant celle de Nantes. Le prélat rémois rappelait notamment les règles qui devaient prévaloir en matière de translation: celle-ci peut intervenir seulement lorsque «la nécessité» et «l'utilité» l'exigent, après un examen attentif du cas et à la suite de la décision d'un synode ou du consentement du pape ${ }^{7}$. Nous ne reviendrons pas en détail sur l'argumentation canonique déployée à cette occasion et étudiée de manière exhaustive par S. Scholz ${ }^{8}$. Le dossier mérite cependant d'être approfondi sur d'autres points, concernant notamment la situation d'Actard à Nantes ou la justification avancée par

4. La Chronique de Nantes (570 environ-1049), éd. R. Merlet, Paris, 1896. L'épiscopat d'Actard est abordé aux p. 39-65.

5. C'était l'opinion du regretté H. GuILLOTEL.

6. Il s'agit des: lettre du concile de Soissons au pape Nicolas $\mathrm{I}^{\mathrm{er}}, 18$ août 866 (J.D. MANSI, Sacrorum conciliorum nova et amplissima collectio, t. 15, Venise, 1770, réimpr. Paris-Leipzig, 1902, col. 732-734 [= Die Konzilien der karolingischen Teilreiche 860-874, éd. W. Hartmann, M.G.H., Concilia, t. 4, Hanovre, 1998, p. 218-221, n² 23, D]); lettres de Charles le Chauve à Nicolas I ${ }^{\mathrm{er}}$, fin octobre 867 (Charles le Chauve, Epistolae, éd. J.P. Migne, P.L., t. 124, col. 870-875, $\mathrm{n}^{\circ} \mathrm{v}$; mention G. Tessier, Recueil des actes de Charles IIle Chauve, roide France, t. 2, Paris, 1952, p. 176-177, nº 305) et à Hadrien II, début septembre 871 (CHARLES LE CHAUVE, Epistolae, col. 876-881, n VII; mention TESSIER, op. cit., t. 2, p. 280-281, $\mathrm{n}^{\circ} 350$ ) ; lettres d'Hadrien II à Charles le Chauve, 23 février 868 (Hadrien II, Epistolae, éd. E. Perels, Epistolae Karolini Aevi, t. 4, p. 704-707, nº 7), 871 [26 décembre] (Ibid., p. 741-743, $\mathrm{n}^{\circ}$ 35), 872 (Ibid., p. 743-746, $\mathrm{n}^{\circ}$ 36), aux évêques du synode de Soissons, février 868 (Ibid., p. 707-709, nº 8), à Actard, 25 février 868 (Ibid., p. 709-710, no 9), à Hincmar de Reims, 8 mars 868 (Ibid., p. 710-712, n 10), à Hérard de Tours, 8 mars 868 (Ibid., p. 713, no 12), au synode de Douzy, 26 décembre 871 (Ibid., p. $\left.738-740, n^{\circ} 34\right)$; lettre du synode de Douzy au pape Hadrien II, 6 septembre 871 (Die Konzilien, p. 523-528); lettres d'Hincmar adressées l'une à Hadrien II, septembre 871 (éd. J.P. Migne, P.L., t. 126, col. 641-648; mention H. SCHRÖRS, Hinkmar, Erzbischof von Reims. Sein Leben und seine Schriften, Fribourg, 1884, réimpr. Hildesheim, 1967, Registrum Hincmari, p. 541, $\mathrm{n}^{\circ} 315$ ) et l'autre en réponse à la consultation d'un évêque non nommé (éd. MiGNE, op. cit., col. 210-230; mention SCHRÖRS, op. cit., p. 542, nº 329, qui date la lettre de 872; FlODOARD, Die Geschichte der Reimser Kirche, éd. M. STRATMANN, M.G.H., SS., t. 36, Hanovre, 1998, p. 361).

7. P.L., t. 126, col. 213: Si autem causa certae necessitatis vel utilitatis exegerit ut quilibet episcopus de civitate in qua ordinatus est ad aliam transferatur, synodali dispositione, vel apostolicae sedis consensione, apertissima ratione manifestum fieri debet [...].

8. S. Scholz, Transmigration und Translation. Studien zum Bistumswechsel der Bischöfe von der Spätantike bis zum Hohen Mittelalter, Cologne-Weimar-Vienne, 1992, p. 130-147. 
l'évêque et son protecteur, Charles le Chauve, pour obtenir la translation du prélat vers une autre Église. Le cas sollicite une attention particulière car il est également invoqué pour démontrer les préoccupations missionnaires du clergé franc à l'égard des Vikings installés dans le royaume franc ${ }^{9}$. En effet, dans sa lettre de 872 , l'archevêque Hincmar reproche à son collègue d'avoir failli à ses devoirs en ne restant pas sur place pour prêcher des païens qui eussent pu être convertis. Toutefois, sorti de son contexte, le propos peut conduire à une interprétation erronée.

\section{La situation d'Actard à Nantes: un prélat menacé?}

Évêque de Nantes depuis 843/844, Actard avait été chassé une première fois par Nominoé, avant de recouvrer son siège quelques années plus tard à la faveur d'un rapprochement entre le roi breton et Charles le Chauve ${ }^{10}$. Dès août 866 , les évêques du concile de Soissons présentent la situation du prélat comme intenable et, dans une lettre adressée à Nicolas $\mathrm{I}^{\mathrm{er}}$ en octobre 867, Charles le Chauve insiste sur les épreuves subies par Actard, «l'exil, les liens, la mer et de sévères périls» et la dévastation de Nantes. La ville, «autrefois très florissante», est «maintenant brûlée et détruite de fond en comble», au point d'être "retournée au désert pendant dix ans ${ }^{11}$ ». Son évêque a été chassé, en raison de la feritas et de la persecutio que lui ont fait subir tant les Normands que les Bretons ${ }^{12}$.

Si la plupart des documents, y compris la lettre adressée par Hincmar à Hadrien II en septembre 871, reviennent régulièrement sur les méfaits des deux gentes, dans le détail les griefs ne sont pas identiques. Seul l'archevêque de Reims, dans sa consultation de 872, attribue explicitement l'expulsion $\mathrm{d}^{\prime}$ Actard à Salomon ${ }^{13}$. Les autres missives, particulièrement celle du concile

9. S. Coupland, The Rod of God's Wrath or the People of God's Wrath? The Carolingian Theology of the Viking Invasions, Journal of Ecclesiastical History, t. 42, 1991, p. 553.

10. Nantes fut prise en 850 par Nominoé, qui démantela une partie des portes et des remparts de la cité, laissant pour ainsi dire la ville ouverte à un moment où la pression scandinave s'intensifiait sur la basse Loire (ChÉdeville, GuILLOtel, op. cit., p. 312-313). Le roi breton établit un intrus, Gislard, sur le siège de Nantes, que recouvra Actard avant 856 (Ibid., p. 291-293, 306-307).

11. Actardum Namneticae quondam sedis venerabilem episcopum, exsilium, vincla [sic], mare, dura pericula passum, sed gratia Dei liberatum. Northmannis nimium Britonibusque vicinum, ac perinde civitate sibi commissa, olim florentissima, nunc exusta et funditus diruta, redacta per decennium cernitur in eremum [...] (CHARLES LE CHAUve, Epistolae, col. 875; Die Konzilien, p. 243).

12. Ibid., p. 220.

13. Postea autem a Salomone tyranno eumdem Actardum a civitate sua expulsum [...] (P.L., t. 126, col. 218). 
de Soissons - la plus précise sur la situation religieuse en Bretagne -, déplorent que les Bretons ont distrait la partie du diocèse de Nantes depuis les murs de la cité et usurpé des biens appartenant à cette Église. À en croire Hadrien II, la «continuelle oppression» des Bretons fit souffrir à Actard des maux «presque pires» que celle «des pirates ${ }^{14} »$.

Ces derniers, constate le pape dans une lettre à Charles le Chauve, ont fait de Nantes non seulement une étape (transitum) mais y ont également installé une base (statio) - allusion probable au camp de Betia -, provoquant $l^{\prime}$ exode de la population en de nombreux lieux ${ }^{15}$. En recoupant cette lettre avec d'autres adressées au même moment (début 868), il ressort qu'Actard a été chassé de son siège par les païens, a subi une longue captivité dont il a été libéré par l'intercession de Dieu et a souffert d'une longue errance loin de son Église, qu'il n'a pas défailli et a même prêché le Christ parmi les «païens» et les «infidèles ${ }^{16}{ }$. Ses mérites et ses souffrances justifient,

14. [...] propter eorundem rabiem barbarorum et continuam Brittonum oppressionem, quibus, heu, nimium vicinus esse nosceris, et pene peius ab eis quam a piratis insecutionem pateris (HADRIEN II, Epistolae, p. 709, no 9).

15. [...] paganis videlicet non solum transitum, sed etiam stationem Ibid. facientibus ac per hoc depopulatis undique locis habitatore carentibus, sublatis etiam a Brittonibus, si quae remanserant, rebus [...] (Ibid., p. 706, $\left.\mathrm{n}^{\circ} 7\right)$. L'abandon de Nantes et la désertion provoquée par l'insécurité due à la présence des Normands sur la basse Loire sont admis par la plupart des spécialistes, voir notamment: CHÉDEville, Guillotel, op. cit., p. 312-313; N.Y. TONNERRE, Naissance de la Bretagne. Géographie historique et structures sociales de la Bretagne méridionale (Nantais et Vannetais) de la fin du VIII à la fin $d u X I^{e}$ siècle, Angers, 1994, p. 275-278. Cette situation était catégoriquement rejetée par A. Du MOYNe DE LA BORDERIE, Histoire de la Bretagne, $2^{\mathrm{e}}$ tir., t. 2, Rennes, 1906 , p. 104-105, qui y voyait «une invention de ce déserteur» (= Actard) et jugeait le prélat comme «un triste évêque, toujours agité, toujours courant, Juif-Errant de l'épiscopat». Pour la localisation de Betia (Bièce, auj. rattaché à Nantes ou l'île de Botty à Bougenais, Loire-Atlantique, cant. Rezé), voir J.C. CASSARD, Les Bretons de Nominoë, Rennes, 2002, p. 80.

16. À Charles le Chauve: [...] qui inter cetera virtutum suarum insigna barbarorum rabiem saepe perpessus Christum confiteri non solum coram hominibus, verum etiam coram paganis et infidelibus non erubuit. Ei ergo, cuiuscumque sint numeri, merito possunt oves Christi committi, qui ipsum Christum etiam inter gladios et vincula diversaque pericula praedicare non rennuit (HADRIEN II, Epistolae, p. 706, $\mathrm{n}^{\circ} 7$ ). Aux évêques du synode de Soissons: Sed quia idem reverendus antistes, ut vestra relatio manifestat, a propria ecclesia diutino iam tempore paganorum persecutione propulsus hac illaque peregre proficiscitur, huc atque illuc multis iam fessus erumnis vagabundus incedit [...] (Ibid., p. 707, nº 8). À Actard: Tibique, frater Hactarde, qui dira fatigatus et diuturna captivitatis miseria quassatus nec inter cuneos barbarorum nec inter enses infidelium deficisti, quin etiam Dei providentia mirabiliter erutus et liberatus circumquaque peregrinando procul a propria ecclesia, procul a notis et proximis, propter eorundem rabiem barbarorum et continuam Brittonum oppressionem [...] curriculis iam multis incedis, decus pallii pro Dei solius amore, quem inter furentium paganorum manus et gladios confessus es, praevidimus concedendum (Ibid., p. 709, no 9). Qui sont les «infidèles» mentionnés dans les textes? Le qualificatif ne 
aux yeux d'Hadrien II, l'octroi à Actard du pallium et l'épithète sanctissimus praesul, employée dans une missive où le pape recommande le prélat à $l^{\prime}$ archevêque Hincmar ${ }^{17}$.

À la demande de Charles le Chauve ${ }^{18}$, le pape décida d'accorder une autre Église à Actard ${ }^{19}$, s'il s'en trouvait une vacante, et de conférer à l'évêque le pallium avec les privilèges juridiques attachés à cette dignité. En aucune manière, cette dernière ne vaudrait pour la cité où Actard serait incardiné, qui ne pourrait revendiquer le titre de métropole ${ }^{20}$, mais l'intention d'Hadrien était manifestement de faciliter le transfert d'Actard sur un siège métropolitain ${ }^{21}$. Un temps accueilli (870/871) dans le diocèse de Thérouanne, dont l'Église était privée de pasteur, le prélat fut élu au siège de Tours, vacant depuis la mort de Hérard (juin 871), puis constitué métropolitain par Hadrien II (26 décembre 871). Actard conservait Nantes, l'union provisoire des deux sièges étant justifiée par la situation dans cette dernière cité22.

paraît pas s'appliquer aux Bretons, qui sont par ailleurs qualifiés de pseudochristiani par Hincmar (P.L., t. 126, col. 641). Doit-on aller jusqu'à conjecturer qu'Actard fut livré par les Normands aux musulmans? La basse vallée de la Loire était une étape pour les Scandinaves se rendant en Espagne ou qui en revenaient (Annales de SaintBertin, éd. F. Grat, J. Vielliard, S. Clémencet, Paris, 1964, p. 89; F. Lot, La Loire, l'Aquitaine et la Seine de 862 à 866. Robert le Fort, Bibliothèque de l'École des chartes, t. 76, 1915, p. 473-510 (rééd. dans F. Lot, Recueil des travaux historiques, t. 2, GenèveParis, 1970, p. 785 n. 3). Hadrien était informé des épreuves de l'évêque de Nantes par plusieurs lettres, dont celles du concile de Soissons et de Charles le Chauve, dont il reprend certaines formulations. Sans doute eut-il aussi une relation faite par Actard lui-même, envoyé à Rome par les synodes de Soissons (866), de Troyes (867) et par Charles le Chauve (Die Konzilien, p. 220; Annales de Saint-Bertin, p. 138, 140; FloDOARD, Die Geschichte der Reimser Kirche, p. 252-253). Les lettres d'Hadrien II parvinrent à Servais, portées par Actard, de retour de Rome au printemps 868 (Annales de Saint-Bertin, p. 143-144). On ne peut exclure que l'évêque ait à dessein noirci sa situation; toutefois tous les témoignages s'accordent pour la décrire comme difficile. Seul Hincmar - tardivement (872) - condamna Actard pour n'être pas resté à Nantes, nous y reviendrons.

17. Hadrien II, Epistolae, p. 711, no 10.

18. Charles le Chauve, Epistolae, col. 870-875.

19. Le pape semble avoir été soucieux de débuter son pontificat en se montrant conciliant avec Charles (ScHOLZ, op. cit., p. 133).

20. Le sens à donner à cette dignité est longuement expliqué dans les lettres d'Hadrien II à Charles le Chauve et surtout à Actard (HAdRIEN II, Epistolae, p. 704-707, 709-710, n ${ }^{\text {os }} 7$ et 9 ).

21. Ibid., p. 706, nº 7; E. LESNE, La hiérarchie épiscopale, provinces, métropolitains, primats en Gaule et en Germanie depuis la réforme de saint Boniface jusqu'à la mort d'Hincmar, 742-882, fasc. 1, Lille-Paris, 1905, p. 94-95.

22. Quodsi Namneticae contigerit ecclesiae ad priorem Christo auxiliante statum redire, nihil officiat ei si haec necessitatis unitio, quam videlicet exigit paganorum vastatio, quominus proprium valeat habere pontificem (HADRIEN II, Epistolae, p. $739, \mathrm{n}^{\circ} 34$ ). En février 868, Hadrien II avait assuré au prélat qu'il conserverait les biens de l'Église de 
La position d'Actard posait un problème juridique et théologique, qui est l'objet principal de la longue consultation rédigée par Hincmar en 872. La difficulté était double: la translation d'un évêque vers un autre siège et le cumul de deux Églises.

La législation conciliaire du Bas-Empire avait statué - de manière restrictive - sur le transfert des évêques et il avait fallu attendre le pontificat de Grégoire le Grand pour que fût admise une dispense en invoquant «la nécessité des temps $\mathrm{s}^{23}$ ». Le principe selon lequel l'évêque était choisi pour une Église à laquelle il devait rester fidèle fut par la suite souvent invoqué, notamment pour interdire les translations motivées par l'ambition de parvenir à un siège épiscopal plus riche ou plus prestigieux. Ce souci de stabilité de l'épiscopat est exprimé par la correspondance des papes du viII siècle et l'accumulation dans les Fausses Décrétales, au milieu du IXe siècle, de textes condamnant des pratiques jugées abusives ${ }^{24}$. L'offensive canonique s'appuyait, entre autres, sur l'image du mariage del'évêque avec son Église ${ }^{25}$, un thème que développe abondamment Hincmar dans sa consultation. Dans sa correspondance, Hadrien II s'était attaché à motiver ses décisions concernant Actard en se reférant à la législation de ses prédécesseurs, en particuler Grégoire le Grand, en $868^{26}$, et du pseudo-Antère, en $871^{27}$. Y était notamment réaffirmé le principe selon lequel non enim transit de civitate in civitatem, qui non suo libitu hoc facit aut ambitu, sed utilitate quadam aut necessitate aliorum hortat ${ }^{28}$. Pour le pape, la situation de l'évêque de Nantes justifiait largement le transfert de ce dernier et l'octroi du pallium; afin que nul ne se méprenne sur les intentions d'Actard, Hadrien entendit couper court à toute suspicion d'ambition, qui eut pu flétrir la réputation du prélat ${ }^{29}$.

Hincmar était parfaitement informé de la situation de son collègue nantais. Les deux hommes étaient présents au synode de Soissons (866), auquel

Nantes épargnés par les Normands et les Bretons (Ibid., p. 710, $\mathrm{n}^{\circ} 10$ ). En attendant, il recommandait peu après à Hérard, archevêque de Tours, de concéder à Actard un monastère (Ibid., p. 713, $\mathrm{n}^{\circ} 12$ ).

23. J.GAudemet, L'Église dans l'Empire romain ( $I V^{e}-V^{e}$ siècles), Paris, 1958, p. 356-363.

24. ID., Église et cité. Histoire du droit canonique, Paris, 1994, p. 213-214.

25. Ibid. et ID., Note sur le symbolisme médiéval. Le mariage de l'évêque, L'Année canonique, t. 22, 1978, p. 71-80 (rééd. dans ID., La société ecclésiastique dans l'Occident médiéval, Londres, $\left.1980, \mathrm{n}^{\circ} \mathrm{x}\right)$.

26. HADRIEN II, Epistolae, p. 708, $\mathrm{n}^{\circ} 8$.

27. Ibid., p. 739, no 34 , qui s'appuie également sur le canon 2 du concile de Nicée et une décrétale de Léon $\mathrm{I}^{\mathrm{er}}$. $\mathrm{C}^{\prime}$ est la première fois qu'une lettre pontificale utilise, en le citant, un texte pseudo-isidorien (ScHOLz, op. cit., p. 112).

28. HADRIEN II, Epistolae, p. 739, n 34.

29. Nec ei minorem quam sua fuerat dari volumus, cum, licet hoc ipse minime postulet - ne videlicet alicuius ambitionis fama notetur - conveniet consequi potiorem [...] (Ibid., p. 706, $\left.\mathrm{n}^{\circ} 7\right)$. 
assistait également Hérard de Tours, métropolitain d'Actard et ami ${ }^{30}$ de l'archevêque de Reims. Hadrien II avait recommandé à Hincmar Actard, qui avait été ensuite accueilli dans un diocèse de la province de Reims. La même lettre du pape (mars 868) ${ }^{31}$ insiste sur les éloges dont avait été gratifié le pasteur de l'Église rémoise, qui appuya en 871 la nomination d'Actard à Tours $^{32}$. Rien ne semble alors présager le réquisitoire dressé par l'archevêque de Reims contre son nouveau collègue quelques mois plus tard, même si, auparavant, il avait été visiblement réticent à lui confier le siège des Morins.

\section{La position de Hincmar: réaffirmer le droit de l'Église}

Dans sa consultation de 872, Hincmar cite et commente longuement les dispositions canoniques relatives aux translations d'évêques, avant de contester la mutatio d'Actard à Tours. Non seulement l'utilitas, pour l'Église tourangelle, ne peut être invoquée ${ }^{33}$, mais aussi le maintien d'Actard sur deux sièges viole la loi ecclésiastique et ne peut être qualifié autrement que d'adultère spirituel $^{34}$. Surtout, l'archevêque de Reims remet en cause le bien-fondé du transfert d'Actard, qu'il n'avait pas contesté l'année précédente, et pas davantage dans l'abondante correspondance portant sur l'exil de l'évêque depuis 866. L'argumentation du prélat repose sur deux points:

1. En s'appuyant sur l'exemple de l'apôtre Paul, qu'Actard aurait dû davantage avoir à l'esprit, l'évêque ne devait pas abandonner sa cité, mais au contraire y prêcher sans relâche. Qui sait si, parmi les païens habitant la cité, il ne s'en trouvait pas de nombreux prédestinés par Dieu à la vie et qui eussent pu être convertis par la prédication ${ }^{35}$ ? La nécessité aurait dû pousser le prélat à rester, d'autant plus que là où se trouvent des chrétiens, ils se doivent d'être sauvés. Hincmar se montre inébranlable dans la conviction qui est la sienne dans le débat sur la prédestination, son refus de considérer les hommes prédestinés au péché, fussent-ils païens. Aussi la faute d'Actard est-elle lourde car il a failli à sa mission spirituelle alors qu'il aurait dû méditer l'exemple de ceux qui sont courageusement restés, comme le patriarche

30. FlodoARD, Die Geschichte der Reimser Kirche, p. 274.

31. Hadrien II, Epistolae, p. 710-712, n 10. Notons par ailleurs qu'Actard est un intermédiaire entre le pape et Hincmar, par exemple au moment de l'affaire du divorce de Lothaire II (FLODOARD, Die Geschichte der Reimser Kirche, p. 273, 312) ou entre le bibliothécaire de l'Église de Rome, Anastase, et l'archevêque rémois (Ibid., p. 323).

32. P.L., t. 126, col. 641-648; FlodOARD, Die Geschichte der Reimser Kirche, p. 275.

33. P.L., t. 126, col. 218.

34. Ibid., col. 226.

35. Ibid., col. 225. 
de Jérusalem, les religieux ou les fidèles de Cordoue et des autres Églises de $l^{\prime}$ Espagne $^{36}$. L'argument de necessitas, utilisé pour justifier le transfert d'un évêque, ne peut être ici avancé car si nécessité il y avait, c'était de rester sur place assurer le salut des chrétiens et de prêcher les païens, qu'on pouvait espérer amener sur la voie de la conversion. L'archevêque de Reims ignore - ou plutôt feint d'ignorer - les épreuves subies par Actard, la constance de sa foi, son mérite à faire connaître le Christ qui avaient, selon les propos du pape Hadrien, valu le pallium au prélat.

2. Ce transfert est d'autant moins admissible que la situation à Nantes n'était pas intenable. Hincmar indique qu'Actard a été chassé de son siège par Salomon, mais n'y revient plus par la suite pour envisager seulement la vie dans la cité occupée par les Normands. Comment admettre qu'un évêque, sans charge de famille, ne puisse vivre parmi les païens comme le fait le comte, pourtant pourvu d'une femme et d'enfants ${ }^{37}$ ? Il était possible, pour le prélat, de résider sur place d'autant qu'il disposait, grâce aux largesses royales, de ressources suffisantes pour assurer son entretien et exercer son ministère.

En présentant la situation nantaise sous un jour plus favorable que ne le laissent entendre les témoignages contemporains, Hincmar plaçait Actard sous le coup d'une législation qui interdisait le transfert pour des raisons telles que l'ambition ou l'intérêt. Actard aurait dû rester à Nantes, les motifs pour lesquels il avait quitté son siège n'étaient pas recevables. En agissant de la sorte, il se comportait non en pasteur mais en mercenarius, qui n'hésite pas à abandonner ses brebis quand survient le loup ${ }^{38} \ldots$

Il est tentant de rechercher dans cette consultation certains traits de caractère de l'archevêque de Reims, son «manque de souplesse en face des "accommodements" humains lorsque les textes canoniques sont en jeu ${ }^{39}$ ", son horreur de la désertion, en particulier celle des clercs qui, installés par Dieu dans leurs fonctions, doivent y vivre et y mener le combat ${ }^{40}$. Le texte porte également la marque des débats - sur la prédestination par exemple - dans lesquels l'auteur est alors pleinement engagé et, peut-être aussi, la trace des désillusions d'Hincmar à l'égard de Charles, auquel il s'oppose dans l'affaire ${ }^{41}$. La réaction tardive (872) de Hincmar à la translation de son collègue a pu aussi être expliquée par un certain opportunisme, qui aurait

36. Ibid., col. 228.

37. Ibid., col. 229. Notons qu'aucun autre comte contemporain n'est attesté par ailleurs à Nantes.

38. Ibid.

39. J. Devisse, Hincmar, archevêque de Reims, 845-882, t. 2, Genève, 1976, p. 789.

40. Ibid., p. 1106-1107.

41. Devisse (Ibid., p. 790) suggérait qu'Hincmar glissait alors «vers l'indifférence retrouvée du roi », qui l'aurait conduit «à une amertume croissante». 
conduit l'archevêque à ménager un protégé du roi tant que n'était pas réglé son différend avec Hincmar de Laon ${ }^{42}$. Il faut pourtant se garder de discréditer trop rapidement son témoignage. Entre le «très saint évêque» et le mauvais pasteur qui abandonne son troupeau, entre le témoin du Christ au milieu des païens et le prélat oublieux de ses devoirs, entre le portrait d'une cité anéantie et l'image d'une ville où il est possible de résider, le dossier révèle des enjeux dont les Normands servent de faire valoir. Politiques et religieux, d'abord: outre l'intérêt, pour Charles le Chauve, de promouvoir un évêque fidèle, il apparaît qu' Actard est un élément du dispositif destiné à faire pièce au schisme breton ${ }^{43}$. La désignation du prélat à Tours n'était pas anodine ${ }^{44}$, et on ne peut écarter que la succession de l'archevêque Hérard ait été préparée dès $867^{45}$. Des enjeux canoniques ensuite, en réaffirmant la primauté du droit de l'Église sur des choix contestables, fussent-ils encouragés par le roi. Dans l'immédiat, Actard dut résigner son siège de Nantes, où fut élu Hermengarius ${ }^{46}$.

\section{Une pratique répandue?}

Quelques années plus tard (876), Hincmar s'en prit à un autre transfert abusif en faveur de Frothaire, archevêque de Bordeaux ${ }^{47}$. Charles le Chauve avait demandé au pape Jean VIII d'autoriser la translation de l'intéressé vers le siège métropolitain de Bourges, dont le titulaire, Wulfade, venait de mourir ${ }^{48}$.

42. Scholz, op. cit., p. 145. De plus, l'archevêque de Reims avait sollicité Actard, avec Raginelmus, évêque de Noyon, et Jean, évêque de Cambrai, pour juger le différend opposant Hincmar de Laon à plusieurs de ses vassaux (FLODOARD, Die Geschichte der Reimser Kirche, p. 296; Hincmar De Reims, Hincmar De LaON, Die Streitschriften, éd. R. SCHIEFFER, Die Konzilien der karolingischen Teilreiche 860-874, Suppl. 2, M.G.H., Concilia, t. 4, Hanovre, 2003, p. 368, Rotula prolixa (août-nov. 870); Die Konzilien, p. 436, no 37 (concile de Douzy, 5 août-6 septembre 871); Actard intervient à différentes reprises lors du concile qui condamne l'évêque de Laon (Ibid., p. 505, 506, 517).

43. Actard peut être tenu pour un prélat réticent au développement du regnum breton (ChÉdevilLe, Guillotel, op. cit., p. 310-311, 331).

44. De fait, elle entraîna une dégradation des rapports entre les Églises bretonnes et la métropole tourangelle (Ibid., p. 331).

45. Il n'est pas exclu que Hérard ait lui-même suggéré Actard pour lui succéder. La Chronique de Nantes (p. 50) insiste sur les relations quasi filiales entre les deux prélats: Herardus vero archiepiscopus, Actardi episcopi prudentia et probitate diligentissime cognita, quasi filium carissimum semper secum familiariter habere voluit.

46. Ibid., p. 65.

47. Annales de Saint-Bertin, p. 202, 204. Sur ce dossier, voir Scholz, op.cit., p. 147-157.

48. La lettre de Charles le Chauve n'est pas conservée, mais il est possible d'en reconstituer la teneur à partir de la missive que Jean VIII envoya à l'empereur en 
La demande était motivée par l'infestatio paganorum ${ }^{49}$ et la correspondance de l'année 876 présente la cité et la province de Bordeaux comme durement éprouvées par les Normands ${ }^{50}$. En réalité, rien n'indique que Bordeaux ait, à ce moment, été particulièrement touchée par les assauts des Vikings. La ville, exposée aux attaques des pirates, avait subi les raids à plusieurs reprises, en $847-848^{51}$, en $855^{52}$, et peut-être en $864^{53}$, mais aucun autre n' est attesté par la suite durant l'épiscopat de Frothaire, archevêque depuis ca $860^{54}$. Plus vraisemblablement, Charles tenait à placer un homme de confiance à la tête de la métropole de Bourges, dont le contrôle lui paraissait indispensable pour affermir son pouvoir en Aquitaine ${ }^{55}$. Frothaire était en effet l'un des fidèles les plus influents del'empereur, qui l'avait comblé de faveurs en Aquitaine ${ }^{56}$. Abbé de Saint-Hilaire de Poitiers en 868, de Saint-Julien de Brioude au moins

octobre 876 (Jean VIII, Registrum, éd. E. Caspar, Epistolae Karolini Aevi, t. 5, M.G.H., Epistolae, t. 7, Berlin, 1928, p. 8-9, n 9).

49. Annales de Saint-Bertin, p. 204: lecta est proclamatio Frotharii Burgdegalensis archiepiscopi, quia non poterat consistere propter infestationem paganorum in ciuitate sua $[\ldots]$.

50. JEAN VIII, Registrum, p. 8-9, no 9: super exterminio Burdegalensis provincie [...]; Ibid., p. 11-12, $\mathrm{n}^{\circ} 13$ (lettre au clergé et au peuple de la cité de Bourges) : idem nostri legati discere potuerunt, ita esse paganorum persecutionibus desolatam [...]; Ibid., p. 12-13, $\mathrm{n}^{\circ} 14$ (lettre aux suffragants de la province de Bourges): sed precipue incursibus Nortmannorum delatam et in solitudinem partim gladiis partimque captivitate deductam $[\ldots]$.

51. Annales de Saint-Bertin, p. 35; Les premières annales de Fontenelle (Chronicon Fontanellense), éd. J. Laporte, Rouen-Paris, 1951, p. 81; Annales Engolismenses, éd. G.H. Pertz, M.G.H., SS., t. 16, Hanovre, 1859, p. 486 ; Chronicon Aquitanum, éd. ID., M.G.H., SS., t. 2, Hanovre, 1828, p. 253.

52. Annales de Saint-Bertin, p. 70.

53. J. CALmette, Le siège de Toulouse par les Normands en 864 et les circonstances qui s'y rattachent, Annales du Midi, t. 29, 1917, p. 153-174.

54. Sur cette datation: J. Nelson, Charles le Chauve, Paris, 1994, p. 216. L. Auzias, L'Aquitaine carolingienne (778-987), Toulouse-Paris, 1937, p.377-378, partant des pièces du dossier sur la translation de Frothaire, admettait que l'archevêque avait quitté la métropole bordelaise aux alentours de 870, en raison des incursions normandes. Le fait était catégoriquement rejeté par W. Vogel, Die Normannen und das fränkische Reich bis zur Gründung der Normandie (799-911), Heidelberg, 1906, p. 250, qui y voyait un prétexte au transfert de Frothaire vers Bourges. J. RenAud, Les Vikings de la Charente à l'assaut de l'Aquitaine, Pau, 2002, p. 47-48, n'aborde pas la question du transfert du prélat mais relève que le passage des Miracles de Saint-Benoît d'Adrevald de Fleury relatif aux assauts des Normands en Aquitaine dans les années 860 ne mentionne aucune attaque contre Bordeaux. Au total, en dehors des pièces concernant la translation, aucun indice certain ne permet de postuler que la ville ait subi alors les assauts des Vikings.

55. Scholz, op. cit., p. 148. Cela avait été le cas une dizaine d'années auparavant, lorsque Charles assura la désignation de Wulfade (NeLSON, op. cit., p. 234).

56. Ibid., p. 234. 
depuis 874 et peut-être de Charroux ${ }^{57}$, le prélat semble avoir un temps administré l'Église de Poitiers ${ }^{58}$, avant de demander sa translation pour Bourges.

L'affaire n'allait pas de soi. Conformément à la règle rappelée par Hincmar en 872 , le cas devait être sérieusement examiné et soumis à l'approbation du synode, en l'occurrence celui réuni à Ponthion en juin/juillet 876 . La cause de Frothaire fut jugée recevable par les envoyés pontificaux ${ }^{59}$, mais Charles essuya un refus du concile ${ }^{60}$, dont les participants avaient d'autre part été indisposés par l'attitude du protégé de l'empereur ${ }^{61}$. Toutefois, dès octobre 876, sans visiblement tenir compte de l'avis de l'assemblée, Jean VIII autorisait la translation ${ }^{62}$, justifiant sa décision par la situation déplorable du siège de Bordeaux et la necessitas inhérente à cette situation ${ }^{63}$. Le transfert n'était cependant nullement définitif et devait prendre fin dès que la nécessité ne s'en ferait plus sentir ${ }^{64}$. En dépit de cela, Frothaire s'accrocha à Bourges, provoquant (ca 884 ) la plainte d'un moine de sa province ${ }^{65}$ et, plus tard, l'intervention du pape Étienne V (885-891), qui ordonna aux évêques $\mathrm{du}$ royaume de forcer l'archevêque récalcitrant à retourner sur son siège bordelais. Frothaire mourut en juin 889, avant que la procédure n'aboutisse.

Les difficultés de Frothaire avaient embarrassé Foulques de Reims, qui s'en était ouvert au roi Carloman II pour lui demander de défendre son collègue de Bourges ${ }^{66}$. Plus tard (895-896), le métropolitain rémois fit valoir auprès du pape Formose, là encore au nom de la necessitas, le transfert de Hériland de Thérouanne, dont l'évêché avait été ravagé par les Normands ${ }^{67}$.

57. L'identité de l'archevêque Frothaire avec son homonyme contemporain abbé de Charroux est admise par G. Tessier, Recueil des actes de Charles II le Chauve, t. 2, p. 332, 334 s., $\mathrm{n}^{\text {os }} 374,375$ ) et NeLSON (op. cit, p. 234), mais considérée avec plus de réserve par SchOLz (op. cit., p. 147).

58. Ibid., p. 149.

59. Peut-être en contrepartie d'un soutien de Charles pour obtenir une condamnation de Formose, évêque de Porto (Ibid., p. 150-151).

60. Annales de Saint-Bertin, p. 204.

61. Charles avait obtenu la désignation d'Anségise de Sens comme vicaire pontifical au nord des Alpes, mais se heurta à des résistances des évêques au moment où il la fit confirmer par le concile, à l'exception de Frothaire, qui donna une réponse conforme aux vœux de l'empereur (Ibid., p. 202).

62. JEAN VIII, Registrum, p. 8-9, $\mathrm{n}^{\circ} 9$.

63. Ibid. et $\mathrm{p}$. $11-13, \mathrm{n}^{\text {os }} 13$ et 14 .

64. Ibid., p. 11-13, n ${ }^{\text {os }} 13$ et 14; FlodOARD, Die Geschichte der Reimser Kirche, p. 367 (lettre du pape Étienne V, vers 887, à l'archevêque de Reims Foulques et d'autres évêques de Gaule).

65. Ibid., p. 364-365.

66. Ibid.

67. Ibid., p. 377: Item pro Herilando Tarunanensi episcopo, cujus episcopio a Normannis depopulato, eum necessitate cogente ad se venientem [...]. Sur ce point nous renvoyons également à Mériaux, Thérouanne et son diocèse, p. 402-403; ID., Parochiae barbari- 
En attendant, l'intéressé fut momentanément établi dans une Église privée de pasteur-Châlons -, jusqu'à ce qu'il fût titulaire du siège. Afin de favoriser son projet, Foulques écrivit à un évêque romain, Pierre, pour que ce dernier intervienne auprès de Formose en vue d'obtenir une réponse positive; il lui suggéra, pour argumenter son propos, de recourir à l'exemple d'Actard ${ }^{68}$. Les motivations réelles, plus spécieuses, avaient quelques raisons d'offenser le droit canonique. D'une part, Foulques invoquait la barbarie des mœurs et de la langue des habitants du diocèse de Thérouanne pour demander qu'on donnât à Hériland un successeur qui leur fût plus approprié: le prétexte cachait mal les difficultés pastorales des évêques de cette Église, en particulier sous l'épiscopat de Hériland ${ }^{69}$. D' autre partet surtout, l' archevêque de Reims cherchait à établir sur le siège de Châlons un évêque favorable à Charles le Simple, son protégé, contre le candidat du roi Eudes, Bertaire, pourtant élu canoniquement par le clergé et le peuple de cette cité$^{70}$. Foulques réussit finalement à imposer un autre clerc, au prix d'une brouille avec la papauté.

Quelques cas ne font pas une généralité mais on peut se demander, pour conclure, dans quelle mesure l'infestatio paganorum n'était pas une raison évoquée pour justifier des dispositions qui répondaient à des motifs moins avouables. D' après les Annales de Fulda, le pape Étienne V aurait, en 886 et sur la demande de Charles le Gros, autorisé la translation vers d'autres évêchés de prélats dont le diocèse avait été ravagé par les païens ${ }^{71}$. En fait, il est très incertain qu'une telle mesure de portée aussi générale ait été décrétée, non seulement en raison de ses contradictions flagrantes avec le droit ecclésiastique, mais aussi parce qu'elle ouvrait la voie à des abus dont nous rendent compte les exemples cités précédemment. Sans doute faut-il y voir la généralisation, par l'annaliste, d'un propos qui concernait au départ seulement des cas bien déterminés. Pour autant, elle témoigne vraisemblablement de la banalisation d'un argument clairement employé à des fins partisanes.

Université de Caen

Pierre BAUDUIN

cae? Quelques remarques sur la perception des diocèses septentrionaux de la Gaule pendant le haut Moyen Âge, Revue du Nord, t. 87, 2005, p. 299; ID., Gallia irradiata, p. 161.

68. Flodoard, Die Geschichte der Reimser Kirche, p. 393.

69. MériauX, Gallia irradiata, p. 160-161.

70. FlodoARD, Die Geschichte der Reimser Kirche, p. 377-378; voir également M. Sot, Un historien et son Église au Xe siècle : Flodoard de Reims, Paris, 1993, p. 191-192.

71. Annales Fuldenses, éd. G.H. Pertz et F. Kurze, M.G.H., SS. rer. Ger. in usum scholarum, t. 7, Hanovre, 1891, p. 114. 